\title{
Energy Transports in Toda Lattices with Quasiperiodic On-site Potentials
}

\author{
Zhenjun Zhang ${ }^{1, a}$, Jing Kang ${ }^{1}$ and Chunmei Tang ${ }^{1}$ \\ ${ }^{1}$ College of Science, Hohai University, 210098 Nanjing, Jiangsu, People's Republic of China
}

\begin{abstract}
We study numerically the process of energy transports in Toda lattices with quasiperiodic on-site potentials. The total energy was initially equidistributed the $10 \%$ of lowest frequency linear modes. For Toda model without on-site potentials, only several new low frequency modes are excited, but the energy equipartition is not achieved. When the quasiperiodic on-site potentials are added, the energy transfers continuously to the high frequency modes and finally evolves towards energy equipartition. We further study the equipartition time $T_{e q}$ as a function of energy density $\varepsilon$ for different nonlinear parameters $\alpha$ and different strengths of on-site potentials $\delta$. In the thermodynamic limit, the dependence of $T_{e q}$ on $\varepsilon$ is found to display a power law behaviour, that is, $T_{e q} \propto \varepsilon^{a}$. The exponenet $a$ is found to be -2.03 and is independent of the values of $\alpha$ and $\delta$.
\end{abstract}

\section{Introduction}

The study of energy transfers in nonlinear lattices has a long history but is still far from been completed. The first numerical calculation was performed by Fermi, Pasta, and Ulam (FPU) with the initial energy in a lowest frequency mode [1]. It was expected the nonlinearity could cause the energy transfers to the high frequency modes, resulting in energy equipartition. However, energy equipartition was not observed. On the contrary, the energy remained concentrated only on some modes. Since then, extensive studies on the energy transport behaviors in nonlinear lattices have been carried out [2-26]. For the FPU model, there is a general agreement that two well separated time scales are present for weak nonlinearity $[15,20-22,24]$. That is, in a relative short time scale, a kind of metastable state is appeared. In this state, in addition to the initial excited states, only several new low frequency modes are excited, but still far from energy equipartition. This is the state observed in the original FPU paper [1]. Such a state, however, is only stationary. On a much longer time scale, the high frequency modes take part in the energy sharing. Finally, the energy equipartition is achieved. The dependence of the equipartition time $T_{e q}$ on the energy density $\varepsilon$ in the thermodynamic limit was recently found to grow as $\varepsilon^{-9 / 4}$ when the initially frequency modes arbitrarily close to zero are excited [21]. Another extensively studied model is the classical lattice $\varphi^{4}$ model. The equipartition time in this model is found to be an exponential law with $1 / \varepsilon$ [5].

On the other hand, the energy transport behaviors of Toda model have been found very similar to the FPU model in the short time scale [21, 22, 24]. But the difference is also obvious. Energy equipartition was not achieved in Toda model, which indicates that the Toda model is integrable and nonergodic [22].

\footnotetext{
${ }^{\mathrm{a}}$ Corresponding author: hi_zhangzhenjun@sina.com
}

In this paper, we are devoted to the energy transport problem in Toda model with the effect of quasiperiodic on-site potentials. The purpose of this paper is to clarify two questions. Firstly, whether can the energy equipartition being achieved with the effect of quasiperiodic on-site potentials. Secondly, if it dose, what is the scaling law for the equipartition time $T_{e q}$ as a function of the energy density $\varepsilon$ in the thermodynamic limit. This paper is organized as follows. In Sec. 2, we outline the model, the calculated physical quantities, and the numerical approach. Numerical results are presented in Sec. 3. We summarize this work in Sec. 4.

\section{Model, physical quantities, and numerical method}

The Hamiltonian of the Toda model with on-site potentials is

$$
H=\sum_{n=1}^{N} \frac{p_{n}^{2}}{2}+\sum_{n=0}^{N} V_{T}\left(q_{n+1}-q_{n}\right)+\frac{1}{2} \sum_{n=1}^{N} V_{n} q_{n}^{2},
$$

where $V_{T}$ has the form

$$
V_{T}\left(q_{n+1}-q_{n}\right)=\frac{1}{4} \alpha^{-2} \cdot\left[e^{2 \alpha\left(q_{n+1}-q_{n}\right)}-1-2 \alpha\left(q_{n+1}-q_{n}\right)\right] .
$$

$p_{n}$ and $q_{n}$ are momentum and the displacement with respect to the equilibrium position of $n$th particle. $N$ is the particle number of the system. $\alpha$ is the nonlinear parameter. $V_{n}$ represents the on-site potentials. For Toda model, the on-site potentials for all $n$ are zero. For quasiperiodic systems, on-site potentials are arranged in quasiperiodic sequences. The quasiperiodic systems represent an intermediate situation between periodic and random systems. One of the simplest prototypes of 
quasiperiodic systems is the Fibonacci lattice [27, 28].It has been rigorously proved [29] that the Fibonacci lattice has a singular continuous spectrum and critical wave functions, which means that the eigenstates are neither localized nor extended. In this paper, $V_{n}$ takes two values $V_{A}$ and $V_{B}$ that are arranged according to Fibonacci sequences. The Fibonacci sequences are defined by the binary substitution rule

$$
A \rightarrow A B, B \rightarrow A
$$

starting with letter $A$. To generate a quasiperiodic lattice we arrange the on-site potentials such that $V_{A}\left(V_{B}\right)$ stands for $A(B)$ in the Fibonacci sequences, with $V_{A}=1+\delta, V_{B}=1$ $\delta$, where $\delta$ represents the strength of on-site potentials. In this paper, we consider the case of fixed boundaries, i.e., $q_{0}=q_{N+1}=0$. In the linear case $(\alpha=0)$, the model of particles can be put in the form of $N$ independent normal modes and is therefore integrable and nonergodic.

For $\alpha=0$, Eq. (1) with $q_{n}=A_{n} e^{i \omega t}$ is reduced to the linear eignvalues problem with

$$
-A_{n+1}-A_{n-1}+\left(2+V_{n}\right) A_{n}=\lambda A_{n} .
$$

The eigenstates $A_{n}^{k}$ are the linear modes. The frequencies $\omega_{k}=\sqrt{\lambda_{k}}$. To each mode $\mathrm{k}$ one can associate a harmonic energy

$$
E_{k}=\frac{1}{2}\left(P_{k}^{2}+\omega_{k}^{2} Q_{k}^{2}\right)
$$

and a phase $\varphi_{k}$ is defined by

$$
P_{k}=\sqrt{2 E_{k}} \cos \left(\varphi_{k}\right), Q_{k}=\sqrt{\frac{2 E_{k}}{\omega_{k}^{2}}} \sin \left(\varphi_{k}\right)
$$

Energy equipartition means that the average of $E_{k}$ up to time $T$, namely

$$
\bar{E}_{k}(T)=\frac{1}{T} \int_{0}^{T} E_{k}(P(t), Q(t)) d t
$$

for large $T$ converges to the energy density $\varepsilon=E / N, E$ denoting the total energy of the system.

On the basis of the $\bar{E}_{k}$ 's, one should then introduce a paremeter providing a distance to equipartition. A frequency used parameter is the "effective number of degrees of freedom"

$$
\xi(t)=\frac{e^{\eta(t)}}{N},
$$

where $\eta$ is the "epectral entropy"

$$
\eta(t)=-\sum_{k=1}^{N} F_{k}(t) \log F_{k}(t), F_{k}(t)=\frac{\bar{E}_{k}(t)}{\sum_{j=1}^{N} \bar{E}_{j}(t)} .
$$

Equipartition denotes to $\xi=1$.

In this paper, the energy was initially equidistributed the $10 \%$ of lowest frequency normal modes, with random initial phases $\varphi_{k}$, uniformly distributed in the interval [0, $2 \pi$. We use a $\mathrm{SABA}_{2} \mathrm{C}$ symplectic algorithm [30] to integrate the equation of motion derived by the Hamiltonian (1). The typical integration time step is $\Delta t=$ 0.1. The corresponding relative error in energy conservation, when all modes are excited and do contribute to the total energy, is around $10^{-5}$. A further decrease of time step by one order of magnitude does not affect the result. In our numerical calculations, we generate a very long but finite Fibonacci chain with size
$M(M>>N)$ according to the substitution rule mentioned above. Then we select a chain with $N$ consecutive on-site potentials, with the starting position chosen randomly. Because of the nonuniform nature of Fibonacci lattices, the $\xi(t)$ differs slightly form chain to chain. For this reason, we present the results of the quantities defined above after first averaging them over ten different selections. We use $\langle\cdot\rangle$ to denote the averaging results. Averages on more than ten different selections are also calculated. The variation on the result is negligible.

\section{Numerical results}

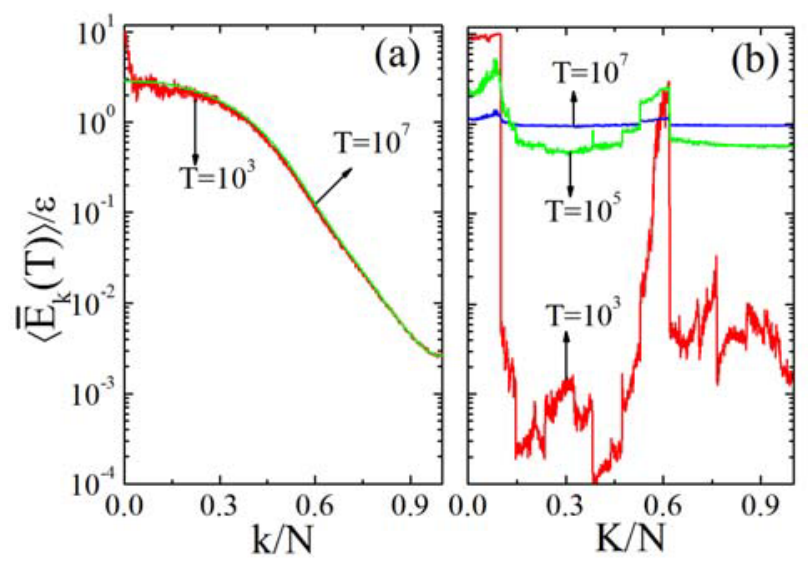

Figure 1. (a) The averaged energy spectrum at different times $\mathrm{T}$ for Toda model without on-site potentials for $\mathrm{N}=1024, \alpha=1.0$, $\varepsilon=0.01$. (b) The averaged energy spectrum at different times $T$

for Toda model with quasiperiodic on-site potentials for $\mathrm{N}=1024, \alpha=1.0, \varepsilon=0.01$, and $\delta=0.5$.

Figure 1(a) shows the results of $\left\langle\bar{E}_{k}(T)\right\rangle / \varepsilon$ versus $\mathrm{k} / \mathrm{N}$ for Toda model without on-site potentials for $\mathrm{N}=1024, \alpha=1.0$, $\varepsilon=0.01$ at different times T. Quite soon, already at $\mathrm{T}=10^{3}$, a well defined profile is formed, in which only some low frequency modes effectively take part in energy sharing. The energies of the remaining ones decay exponentially with $\mathrm{k} / \mathrm{N}$. Since then, the energy profile keeps its form nearly unchanged. Energy equipartition can not be achieved for Toda model without on-site potentials. It proves that the Toda model is integrable and nonergodic. Figure 1(b) shows the results of $\left\langle\bar{E}_{k}(T)\right\rangle / \varepsilon$ versus $\mathrm{k} / \mathrm{N}$ for Toda model with quasiperiodic on-site potentials for $\mathrm{N}=1024, \alpha=1.0, \varepsilon=0.01$, and $\delta=0.5$ at different times T. It can be seen that the energy of the initial excited modes decreases continuously with time. Meanwhile, the energy of other frequency modes grows continuously with time. Finally, the energy of all modes approaches to the global equipartition. It indicates that the integrable property is destroyed and the energy equipartition is therefore can be achieved with the effect of quasiperiodic on-site potentials. 


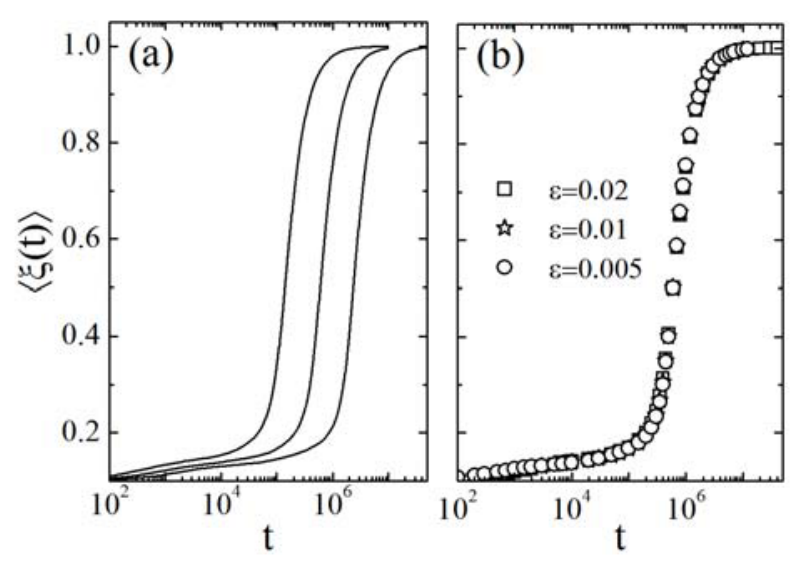

Figure 2. (a) The dependence of $<\xi(t)>$ on time $t$ for $N=1024$, $\delta=0.5, \alpha=1.0$ and different values of $\varepsilon$ in semi-log scale. The curves from left to right at $\langle\xi(t)>=0.5$ are for $\varepsilon=0.02,0.01,0.005$, respectively. (b) The results of $\langle\xi(t)>$ for different $\varepsilon$ are horizontally translated, in such a way the coincide at $\langle\xi(t)\rangle=0.5$ (the one at $\varepsilon=0.01$ is kept fixed).

In order to obtain the overall process of energy transport, we study the properties of $<\xi(t)>$. The results of $<\xi(\mathrm{t})>$ at different $\varepsilon$ with $\mathrm{N}=1024, \alpha=1.0$ and $\delta=0.5$ in semi-log scale are shown in Fig. 2(a). It is seen that on a sufficiently large time scale, all values of $\langle\xi(\mathrm{t})\rangle$ grow from 0 to 1 , with very similar sigmoidal profiles. It illustrates that the energy equipartition is sure to achieve on a sufficiently large time scale. When decreasing the value of $\varepsilon$, the time required to reach equipartition increases. Now, we turn to the definition of the equipartition time $T_{e q}$. We choose the time when $<\xi(\mathrm{t})>$ reaches a value $\xi_{\text {eq }}$ as the equipartition time $T_{e q}$. In this paper, we choose $\xi_{\text {eq }}=0.5$, as has been done in Ref. [21]. Obviously, the choice of $\xi_{\text {eq }}$ is very artificial. We find that $T_{e q}$ is quite insensitive to the choice of $\xi_{\text {eq. }}$. This can be seen in Fig. 2(b) where the sigmoidal profiles from Fig. 2(a) are horizontally shifted to coincide at $<\xi(\mathrm{t})\rangle=0.5$.

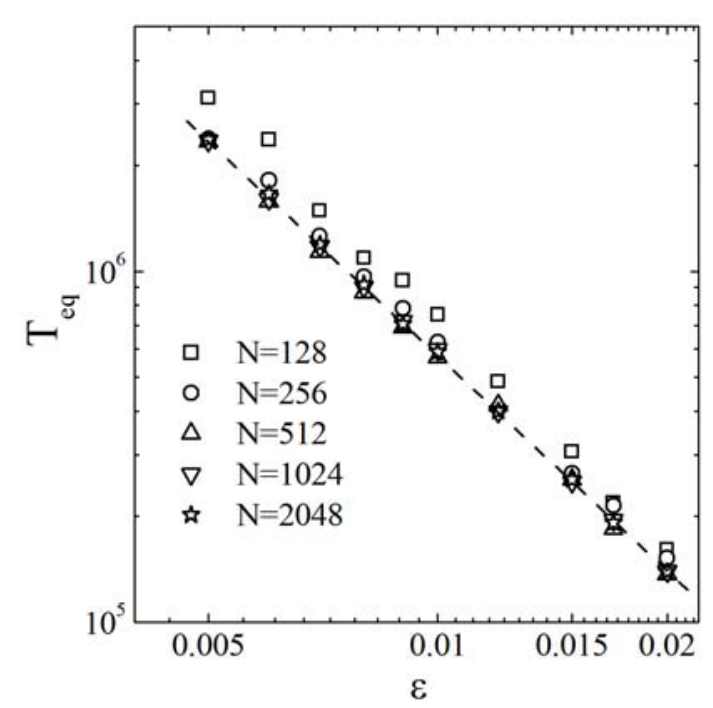

Figure 3. The equipartition time $\mathrm{T}_{\mathrm{eq}}$ as a function of $\varepsilon$ in $\log -\log$ scale, with $\mathrm{N}=128,256,512,1024,2048$ and fixed $\alpha=1.0, \delta=0.5$. The long dashed line has a slope of -2.03 .
In the following, we study the equipartition time $T_{e q}$ as a function of energy density $\varepsilon$ for different nonlinear parameters $\alpha$ and different strengths of on-site potentials $\delta$. Figure 3 shows the results of $T_{e q}$ vs. $\varepsilon$ in log-log scale, for different values of $\mathrm{N}$, namely $\mathrm{N}=128,256,512,1024$, 2048 , at fixed $\alpha=1.0, \delta=0.5$. In the explored range of $\varepsilon$, $T_{e q}$ is found to be nearly independent of $\mathrm{N}$ when $\mathrm{N}$ is large. $\mathrm{N}=512$ seems large enough that the thermodynamics limit is practically achieved. Moreover, $T_{e q}$ versus $\varepsilon$ in the thermodynamics limit displays a power law behavior, that is,

$$
T_{e q} \propto \varepsilon^{a} .
$$

The computed value of the exponent is $\mathrm{a}=-2.03$, which is a little different from that of the FPU model. It illuminates that more time is required to approach equipartition when $\varepsilon$ is decreased. It is known that the energy transfer from the initial excited modes to other modes is induced by the effect of nonlinearity. Less time is needed to approach equipartition when the strength of nonlinearity is increased. When increasing $\varepsilon$, both the energies in linear and nonlinear parts are raised. While the linear terms are quadratic, the nonlinear terms are greater than quadratic. Therefore, the proportion of the energy in nonlinear parts increases i.e., the strength of nonlinearity increases as $\varepsilon$ is increased. Therefore, less time is needed to approach equipartition for larger $\varepsilon$ and the exponent of $\varepsilon$ is thereby less than zero. Besides, we also study the dependencies of $T_{e q}$ on $\varepsilon$ for different values of $\alpha$. The results of $T_{e q}$ as functions of $\varepsilon$ in $\log -\log$ scale, with $\mathrm{N}=1024, \delta=0.5$ and different values of $\alpha$ are shown in Fig. 4. It is seen that $T_{e q}$ versus $\varepsilon$ for different $\alpha$ all display power law behaviors. Moreover, the exponent of $\varepsilon$ is nearly independent of the values of $\alpha$. For fixed $\varepsilon$, it is found that more time is required to approach energy equipartition when $\alpha$ is decreased. The reason is that $\alpha$ is the nonlinear parameter. The strength of nonlinearity increases as the value of $\alpha$ is increased. Therefore, less time is needed to approach equipartition for larger $\alpha$.

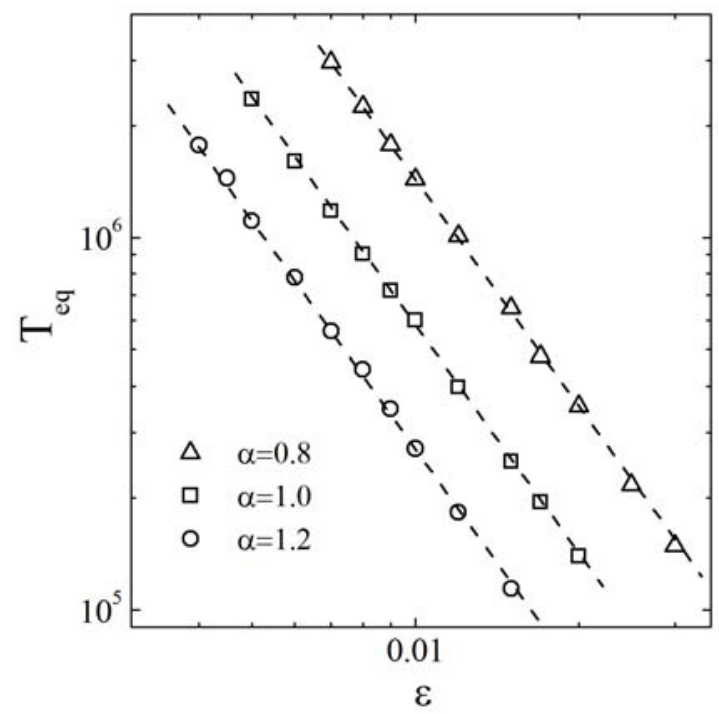

Figure 4. The equipartition time $\mathrm{T}_{\mathrm{eq}}$ as functions of $\varepsilon$ in $\log -\log$ scale, with $\mathrm{N}=1024, \delta=0.5$ and different values of $\alpha$. The slope of the long dashed lines is -2.03 . 
Finally, we study the dependencies of $T_{e q}$ on $\varepsilon$ for different strengths of on-site potentials in the thermodynamic limit. The results of the equipartition time $T_{e q}$ as functions of $\varepsilon$ in $\log -\log$ scale, with $\mathrm{N}=1024, \alpha=1.0$ and different values of $\delta$ are shown in Fig. 5. It is seen that $T_{e q}$ versus $\varepsilon$ for different $\delta$ all display power law behaviors. Moreover, the exponent of $\varepsilon$ is nearly independent of the values of $\delta$. For fixed $\varepsilon$, less time is required to approach energy equipartition when the strength of on-site potentials is increased. It indicates that the strong on-site potentials can accelerate the process to energy equipartition.

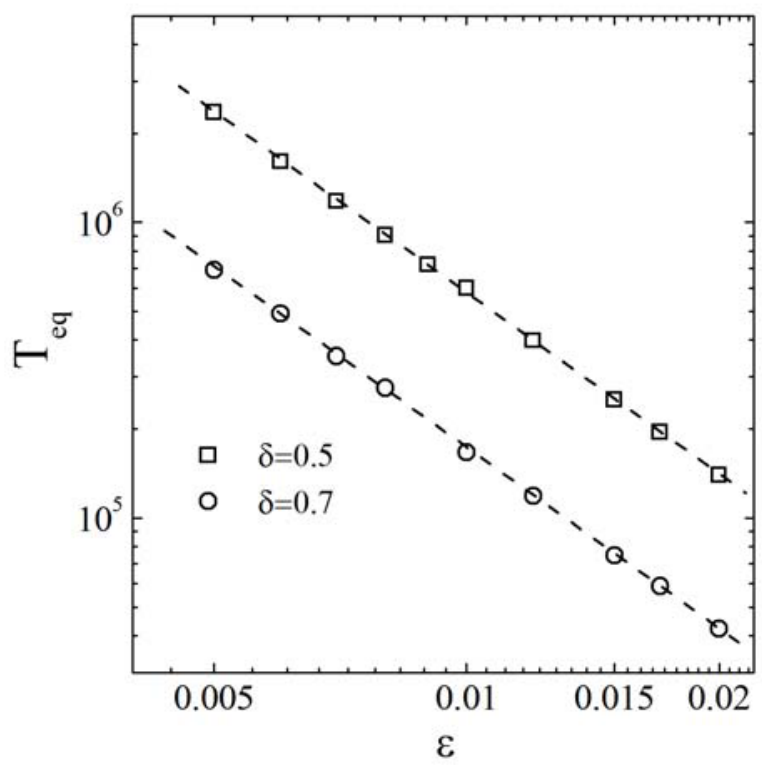

Figure 5. The equipartition time $\mathrm{T}_{\mathrm{eq}}$ as functions of $\varepsilon$ in $\log -\log$ scale, with $\mathrm{N}=1024, \alpha=1.0$ and different values of $\delta$. The slope of the long dashed lines is -2.03 .

\section{Conclusions}

In this paper, we considered the energy transports and time scale to equipartition in Toda lattices with quasiperiodic on-site potentials. At first, the energy was equidistributed the $10 \%$ of lowest frequency modes. For Toda model without on-site potentials, only several new low frequency modes are excited. The energies of the remaining ones decay exponentially with $\mathrm{k} / \mathrm{N}$. Energy equipartition can not be achieved. It proves that the Toda model is integrable and nonergodic. When the quasiperiodic on-site potentials are added, the energy transfers continuously to the high frequency modes and finally evolves towards energy equipartition. It indicates that the integrable property is destroyed with the effect of quasiperiodic on-site potentials. For fixed $\alpha$ and $\delta$, the dependence of $T_{e q}$ on $\varepsilon$ in the thermodynamics limit is $T_{e q} \propto \varepsilon^{a}$. The exponent $a$ is found to be -2.03 , which turns out to be independent of the values of $\alpha$ and $\delta$ and is a little different from that of the FPU model. For fixed $\varepsilon$, it is found that more time is required to approach energy equipartition when $\alpha$ is decreased. The reason is that $\alpha$ is the nonlinear parameter. The strength of nonlinearity increases as the value of $\alpha$ is increased. Therefore, less time is needed to approach equipartition for larger $\alpha$. Besides, less time is required to approach energy equipartition when the strength of on-site potentials is increased, which indicates that the strong on-site potentials can accelerate the process to energy equipartition.

\section{Acknowledgments}

This work is supported by the National Science Foundation of China (Grant No. 11305045), the Special Foundation for theoretical physics Research Program of China (Grant No. 1134711), and the Fundamental Research Funds for the Central Universities of China (Grant Nos. 2013B00414, 2013B00314).

\section{References}

1. E. Fermi, J. Pasta, and S. Ulam, in Collected Papers of E. Fermi, edited by E. Segré (University of Chicago, Chicago, 1965), Vol. 2, p. 978.

2. N. J. Zabusky, and M. D. Kruskal, Phys. Rev. Lett. 15, 240 (1965).

3. R. Livi, M. Pettini, S. Ruffo, M. Sparpaglione, and A.Vulpiani, Phys. Rev. A 28, 3544 (1983).

4. R. Livi, M. Pettini, S. Ruffo, M. Sparpaglione, and A.Vulpiani, Phys. Rev. A 31, 1039 (1985).

5. M. Pettini and M. Landolfi, Phys. Rev. A 41, 768 (1990).

6. M. Pettini and M. Cerruti-Sola, Phys. Rev. A 44, 975 (1991).

7. H. Kantz, R. Livi, and S. Ruffo, J. Stat. Phys. 76, 627 (1994).

8. J. De Luca, A. J. Lichtenberg, and S. Ruffo, Phys. Rev. E 51, 2877 (1995).

9. L. Casetti, M. Cerruti-Sola, M. Pettini, and E. D. G. Cohen, Phys. Rev. E 55, 6566 (1997).

10. J. De Luca, A. J. Lichtenberg, and S. Ruffo, Phys. Rev. E 60, 3781 (1999).

11. K. Ullman, A. J. Lichtenberg, and G. Corse, Phys. Rev. E 61, 2471 (2000).

12. A. Ponno, L. Galgani, and F. Guerra, Phys. Rev. E 61, 7081(2000).

13. P. Villain and M. Lewenstein, Phys. Rev. A 62, 043601(2000).

14. J. De Luca, A. J. Lichtenberg, Phys. Rev. E 66, 026206(2002).

15. L. Berchialla, A. Giorgilli, and S. Paleari, Phys. Lett. A 321, 167 (2004).

16. D. K. Campbell, P. Rosenau, and G. M. Zaslavsky, Chaos 15, 015101(2005).

17. G. P. Berman, F. M. Izrailev, Chaos 15, 015104 (2005).

18. A. J. Lichtenberg, V. V. Mirnov, and C. Day, Chaos, 15, 015109 (2005).

19. A. Carati, L. Galgani, A. Giorgilli, and S. Paleari, Phys. Rev. E 76022104 (2007).

20. G. Benettin, R. Livi, and A. Ponno, J. Stat. Phys. 135, 873 (2009). 
21. G. Benettin and A. Ponno, J. Stat. Phys. 144, 793 (2011).

22. A. Ponno, H. Christodoulidi, Ch. Skokos, and S. Flach, Chaos 21, 43127 (2011).

23. T. Genta, A. Giorgilli, S. Paleari, and T. Penati, Phys. Lett. A 376, 2038 (2012).

24. G. Benettin, H. Christodoulidi, and A. Ponno, J. Stat. Phys. 152, 195 (2013).

25. A. Maiocchi, D. Bambusi, and A. Carati, J. Stat. Phys. 155, 300 (2014).

26. H. J. Matsuyama and T. Konishi, Phys. Rev. E 92, 022917 (2015).

27. M. Kohmoto, L. P. Kadanoff, and C. Tang, Phys. Rev. Lett. 50, 1870 (1983).

28. S. Ostlund, R. Pandit, D. Rand, H. J. Schellnhuber, and E. D. Siggia, Phys. Rev. Lett. 50, 1873 (1983).

29. A. Sütö, Commun. Math. Phys. 111, 409 (1987).

30. Ch. Skokos and E. Gerlach, Phys. Rev. E 82, 036704 (2010) 